\section{Darmkrebs: nach Fatigue und Depression fahnden}

Bei Darmkrebspatienten wird zu wenig auf psychische Probleme eingegangen. Niederländische Forscher wiesen noch Jahre nach der Diagnose ein deutlich erhöhtes Risiko für Fatigue und depressive Symptome nach.

V on über 3.700 Patienten mit kolorektalem Karzinom (CRC) litten fast $40 \%$ unter Fatigue-Symptomen. Die höchsten Werte auf einer 50-PunkteSkala (Fatigue Assessment Scale, FAS) hatten Patienten, bei denen weniger als fünf Jahre seit Diagnosestellung vergangen waren; sie kamen auf durchschnittlich 21 Punkte (höhere Werte stehen für schwerere Symptome). Patienten, bei denen die Diagnose bereits fünf oder mehr Jahre zurücklag, erreichten im Durchschnitt immer noch 20 Punkte, ein gesundes Vergleichskollektiv 18 Punkte. Die Fatigue-Prävalenz in der Durchschnittsbevölkerung war mit $22 \%$ zwar relativ hoch, das Risiko bei den CRC-Patienten jedoch mit $39 \%$ signifikant gröBer $(\mathrm{p}<0,0001)$.

Eine an die Darmkrebs-Operation anschließende Radiochemotherapie war ein unabhängiger Risikofaktor für die Entwicklung einer Fatigue (Odds Ratio 1,63, 95\%-Konfidenzintervall [95\%-KI] 1,17-
2,29). Bei Angst und depressiven Symptomen erhöhte sich das Fatigue-Risiko ebenfalls, und zwar um den Faktor 1,16 (95\%-KI 1,12-1,19) bzw. 1,38 (95\%-KI $1,33-1,43)$. Bei etwa $20 \%$ der Krebspatienten zeigten sich im HAD (Hospital Anxiety and Depression)-Score klinisch relevante Probleme (Angst oder Depression).

Von den Patienten, die in den ersten fünf Jahren in einem Selbstauskunftsbogen (Self-Administered Comorbidity Questionnaire, SCQ) über depressive Symptome berichteten, hätten allerdings nur $57 \%$ eine antidepressive Therapie erhalten, kritisieren die Forscher um Melissa Thong von der Universität Tilburg, Niederlande. In der Vergleichsgruppe wurden dagegen $75 \%$ therapiert.

Bemerkenswert für die Wissenschaftler war auch der hohe Fatigue-Anteil bei Patienten, die vor ihrem Darmkrebs an anderen Tumoren, beispielsweise einem malignen Melanom, gelitten hatten. Von diesen hatten $40 \%$ noch Jahre nach der letzten Krebsdiagnose unter den Erschöpfungserscheinungen zu leiden.

Fazit: Darmkrebspatienten müssen verstärkt auf die möglichen Langzeitfolgen einer Chemotherapie hingewiesen werden. Vor allem auf die psychischen Probleme dieser Patienten sollte stärker eingegangen werden, fordern Thong und Kollegen. Die Ergebnisse ihrer Studie legen nahe, dass sowohl bei der Diagnostik als auch bei der Therapie erhebliche Defizite bestehen.

Elke Oberhofer

Thong MSY et al. Quantifying fatigue in (longterm) colorectal cancer survivors: A study from the population-based Patient Reported Outcomes Following Initial

treatment and Long term Evaluation of Survivorship registry. Eur J Cancer. 2013; 49(8):1957-66.

Darauf sollte man achten: Viele Patienten mit kolorektalen Tumoren fühlen sich noch nach Jahren erschöpft und ausgelaugt.

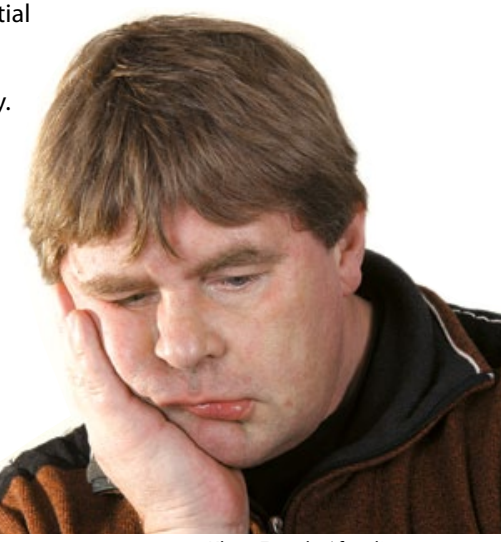

๑ Klaus Eppele / fotolia.com

\section{Therapie nach der Uhr für den Mann bei mCRC}

\section{Molekulare zirkadiane Rhythmen können die Effektivität und Tolerabilität einer Chemotherapie beeinflussen. Beim metastasierten kolorektalen Karzinom ( $\mathrm{mCRC}$ ) profitieren aber nicht alle von einer Therapie, die darauf Rücksicht nimmt.}

G ene beeinflussen eine Vielzahl körpereigener zirkadianer Rhythmen, darunter Zellzyklus, Bioaktivierung und Abbau von Wirkstoffen und ihrer Zielstrukturen. Daher liegt es nahe, den Einfluss einer auf die Chronobiologie abgestimmten Chemotherapie auf den Behandlungserfolg $\mathrm{zu}$ untersuchen. Nachdem eine erste Studie bei metastasiertem kolorektalen Karzinom (mCRC) eine Verlängerung des Überlebens durch chronomodulierte Gabe von 5-Fluorouracil [5-FU] und Oxaliplatin (chronoFLO) gegenüber einer konventionellen Gabe (CONV) gezeigt hatte, blieben zwei Folgestudien ohne eindeutiges Ergebnis. Die Analyse der Daten aus einer Studie, die chronoFLO mit FOLFOX2 verglich, ließ aber einen unterschiedlichen Effekt bei Männern und Frauen vermuten, dem jetzt in einer Metaanalyse aller drei Phase-III-Studien noch einmal nachgegangen wurde. 345 weibliche und 497 männliche Patienten wurden bis zu über neun Jahre nachbeobachtet.

Das Gesamtüberleben als primärer Endpunkt der Metaanalyse zeigte bei Männern mit median 20,8 Monaten einen signifikanten Vorteil der chronomodulierten Therapie gegenüber dem kon- ventionellen Therapieschema (17,5 Monate; $p=0,009)$. Bei Frauen war das chronoFLO-Schema dagegen eher etwas schlechter (median 16,6 vs. 18,4 Monate; $\mathrm{p}=0,012)$.

Fazit: Nur Männer mit mCRC überleben bei einer chronomodulierten Therapie mit 5-FU und Oxaliplatin signifikant länger als bei konventionell verabreichter Chemotherapie mit diesen Substanzen. Die Ergebnisse stützen die weitere Untersuchung einer solchen chronomodulierten Gabe der Chemotherapie in der Erstlinienbehandlung bei Männern. Bei Frauen wird weiter nach der optimalen Gabe der Chemotherapie gesucht, z. B. in Abhängigkeit verschiedener zirkadianer Biomarker.

Friederike Klein

Giacchetti S et al. Sex moderates circadian chemotherapy effects on survival of patients with metastatic colorectal cancer: a meta-analysis. Ann Oncol. 2012;23(12):3110-6. 